\title{
Streptolysin O Antibody Measurement
}

National Cancer Institute

\section{Source}

National Cancer Institute. Streptolysin O Antibody Measurement. NCI Thesaurus. Code C92282.

The determination of the amount of streptolysin $\mathrm{O}$ antibody present in a sample. 